\title{
Exact solutions to the nonlinear equation in traffic congestion
}

\author{
Cheng $\mathrm{Li}^{i^{*}}{ }^{\mathbb{D}}$, Damin Cao' and Qing Du’
}

"Correspondence:

Lcheng8066@126.com

'Shanghai University of Engineering

Science, Shanghai, China

\section{Springer}

\begin{abstract}
In this paper, the KdV-mKdV equation is obtained via the reductive perturbation method which can be applied to model the traffic flow. To overcome the shortcomings of the traditional $\mathrm{KdV}$ - $\mathrm{mKdV}$ equation, the original equation is converted into a space-time fractional equation, which is decreased to a common differential equation by using fractional complex transformation. All possible exact solutions are given through the entire discrimination gadget for polynomial method. In particular, the corresponding options are resembled for the specific parameters to show that each answer in the classification can be realized. And the numerical simulations in the paper additionally confirm this conclusion.
\end{abstract}

Keywords: Exact solution; Traffic flow; KdV-mKdV; Space-time fractional equation; Traveling wave solutions

\section{Introduction}

With the socio-economic development, traffic activities are growing more rapidly. In some specific research, scholars proposed many models to describe, demonstrate and study various complex traffic phenomena [1,2]. Researchers usually regard traffic congestion as a physical phenomenon [3-10]. However, traffic congestion often occurs in the process of traffic operation, which brings great inconvenience to people's travel, among which the car-following model is of great significance and can be used to explain many complex physical phenomena in the traffic flow. Bando [5] proposed an OV model to depict the dynamic behavior of vehicles on a single crowded lane. However, compared with the actual data, it is found that the OV model will exhibit excessive acceleration and unrealistic deceleration. So Peng [7] et al. established the OVD model:

$$
\frac{d^{2} x_{n}(t)}{d t^{2}}=\alpha\left[V\left(\Delta x_{n}(t)\right)-\frac{x_{n}(t)}{d t}\right]+\lambda \Delta v_{n}(t)+\gamma\left[V\left(\Delta_{n+1}(t)\right)-V\left(\Delta x_{n}(t)\right)\right]
$$

where $x_{n}(t)$ represents the position of the $n$th car at time $t, \frac{d x_{n}(t)}{d t}=v_{n}(t)$ represents the velocity of the $n$th car at time $t . \Delta x_{n}(t)=x_{n+1}(t)-x_{n}(t)$ denotes the space headway between the preceding vehicle $n+1$ and the following vehicle $n$, and $\Delta v_{n}(t)=v_{n+1}(t)-v_{n}(t)$ represents the velocity difference between the preceding vehicle $n+1$ and the following vehicle $n . \alpha>0$ indicates the driver's sensitivity coefficient. $\lambda$ indicates the corresponding

(c) The Author(s) 2020. This article is licensed under a Creative Commons Attribution 4.0 International License, which permits use, sharing, adaptation, distribution and reproduction in any medium or format, as long as you give appropriate credit to the original author(s) and the source, provide a link to the Creative Commons licence, and indicate if changes were made. The images or other third party material in this article are included in the article's Creative Commons licence, unless indicated otherwise in a credit line to the material. If material is not included in the article's Creative Commons licence and your intended use is not permitted by statutory regulation or exceeds the permitted use, you will need to obtain permission directly from the copyright holder. To view a copy of this licence, visit http://creativecommons.org/licenses/by/4.0/. 
parameter of velocity difference $\Delta v_{n}(t) . V\left(\Delta x_{n}(t)\right)$ denotes an optimal velocity function. $\gamma$ expresses the reaction parameter of the optimal velocity difference. From all the above we can see that formula (1) does not include the delay of driver's response, which means the driver's stimulation to the preceding vehicle is instantaneous.

Zhu, Dai [8] and Zheng et al. [11] have numerically studied OV site traffic models inside the unstable zone, in which they preset the periodic boundary and analyzed the long-time behavior. Results showed that printed solutions indicative of $\mathrm{mKdV}$ dynamics as kink-like waves appeared. Moreover, Li et al. [12] performed numerical simulations over long time intervals that described a two-lane system with periodic boundaries. This mannequin was modified into a perturbed $\mathrm{mKdV}$ equation next to the critical point. The numerical outcomes corresponding to this region revealed steady periodic traveling wave solutions with consistent amplitude, namely height and period. Hence, these numerical findings provide secure periodic options to the OV visitors gadget do propagate within this unstable region. Based on the above formula (1), Zhou [13] pointed out that there are three typical regions: the area beneath the impartial stability curve is not stable; the region above the coexistence curve is stable; and the region between the two is the metastable zone. The two areas can just be explained through the KdV equation [13] (metastable region) and $m K d V$ equation [14] (unstable region). Combining KdV equation and $m K d V$ equation, we get the $\mathrm{KdV}$-mKdV equation

$$
u_{t}+\mu u u_{x}+\delta u^{2} u_{x}+u_{x x x}=0
$$

where $\mu$ and $\delta$ are constants.

In this paper, in order to make our traveling wave solution more universal, we transform formula (2) into the following space-time fractional equation:

$$
D_{t}^{\alpha} u+\mu u D_{x}^{\alpha} u+\delta u^{2} D_{x}^{\alpha} u+D_{x}^{3 \alpha} u=0
$$

Fractional calculus has a vital role in different areas of science, which has attracted increasing attention due to its nonlocal properties and effective performance on simulating anomalous diffusion, which occurs in transport dynamics in complex systems [15]. Generally, fractional calculus is recognized as one of the best ways to model anomalous diffusion, as observed in plasmas [16].

In order to obtain the exact solutions, many useful methods have been proposed such as the nonlinear steepest descent method [17], collocation method [18], direct method [19], the tanh-sech method [20], sine-cosine method [21, 22], symmetrical method [23, 24], Hirota bilinear method $[25,26]$, and so on. Nowadays, a powerful method named the complete discrimination system for polynomial method has been proposed to obtain the classification of single traveling wave solutions to a series of nonlinear differential equations [27-32]. To our knowledge, the case of Riccati equation is also very important, and we would study this in the future [33]. 


\section{Basic theory}

\subsection{The conformal fractional derivative}

According to Ref. [34, 35], the conformal fractional derivative is defined by

$$
D_{x}^{\alpha} f(x)= \begin{cases}\frac{1}{\Gamma(1-\alpha)} \frac{d}{d x} \int_{0}^{x}(x-t)^{-\alpha}(f(t)-f(0)) d t, & 0<\alpha<1, \\ \left(f^{n}(x)^{\alpha-n}\right), & n \leq \alpha \leq n+1, n \geq 1,\end{cases}
$$

where $\Gamma(x)$ is the gamma function which is defined as

$$
\Gamma(x)=\int_{0}^{x} e^{-t} t^{x-1} d x
$$

There are some properties of the conformal fractional derivative

$$
D_{x}^{\alpha} x^{\gamma}=\frac{\Gamma(1+\gamma)}{\Gamma(1+\gamma-\alpha)} x^{\gamma-\alpha}
$$

While numerical and analytical solutions are obtained, the exact traveling wave solutions to formula (3) are not all-inclusive. As far as we know, the solutions by the complete discrimination system for polynomial technique have not been studied in any other papers.

\subsection{Research method}

By taking the following transformation:

$$
u(x, t)=\Upsilon(\theta), \quad \theta=\frac{\kappa}{\Gamma(1+\alpha)} x^{\alpha}-\frac{c}{\Gamma(1+\alpha)} t^{\alpha}-x_{0}
$$

where $\kappa$ and $c$ represent nonzero constants. And then the nonlinear fractional partial differential equation can be converted into

$$
\psi\left(t, x, \kappa, \omega, u, u^{\prime}, u^{\prime \prime} \cdots\right)=0
$$

According to formula (5), we set

$$
\left(u^{\prime}\right)^{2}=F(u)
$$

where $F(u)$ might be rational function, polynomial function, and any other kind of irrational function. By integrating formula (6), the integral form of the original equation is given by

$$
\pm\left(\theta-\theta_{0}\right)=\int \frac{d u}{\sqrt{F_{n}(u)}}
$$

where $\theta_{0}$ is an integral constant. The main steps of this method are explained above, and many important results have been obtained via this method [24, 25, 28-31]. Also, the considered expansion is a special case of the expansion in the transformed rational function method to solve standard differential equations [36]. 


\section{All exact solutions to space-time fractional KdV-mKdV equation}

According to Ref. [37], substituting the result of (4) into formula (3) yields

$$
-c u^{\prime}+\mu \kappa u u^{\prime}+\delta \kappa u^{2} u^{\prime}+\kappa^{3} u^{\prime \prime \prime}=0 .
$$

By integrating formula (8) with respect to $\theta$, we obtain

$$
-c u+\frac{1}{2} \mu \kappa u^{2}+\frac{1}{3} \delta \kappa u^{3}+\kappa^{3} u^{\prime \prime}=c_{0} .
$$

Multiplying formula (9) on both sides by $u^{\prime}$ and integrating them with respect to $\theta$ again, we get

$$
-\frac{1}{2} c u^{2}+\frac{1}{6} \mu \kappa u^{3}+\frac{1}{12} \delta \kappa u^{4}+\frac{1}{2} \kappa^{3}\left(u^{\prime}\right)^{2}=c_{0} u+c_{1},
$$

where $c_{0}$ and $c_{1}$ are arbitrary integral constants. Furthermore, we can attain

$$
\left(\Upsilon^{\prime}\right)^{2}=-\frac{\delta}{\kappa^{2}} \Upsilon^{4}-\frac{\mu}{3 \kappa^{2}} \Upsilon^{3}+\frac{c}{\kappa^{3}} \Upsilon^{2}+\frac{2 c_{0}}{\kappa^{3}} \Upsilon+\frac{2 c_{1}}{\kappa^{3}} .
$$

Setting $a_{4}=-\frac{\delta}{\kappa^{2}}, a_{3}=-\frac{\mu}{3 \kappa^{2}}, a_{2}=\frac{c}{\kappa^{3}}, a_{1}=\frac{2 c_{0}}{\kappa^{3}}, a_{0}=\frac{2 c_{1}}{\kappa^{3}}$, we can have

$$
\left(\Upsilon^{\prime}\right)^{2}=a_{4} \Upsilon^{4}+a_{3} \Upsilon^{3}+a_{2} \Upsilon^{2}+a_{1} \Upsilon+a_{0}
$$

Making

$$
b_{1}=\frac{\mu}{6 \delta}, \quad \Phi=\left(a_{4}\right)^{\frac{1}{4}}\left(\phi+\frac{b_{1}}{2}\right), \quad \theta_{1}=\left(a_{4}\right)^{\frac{1}{4}} \theta,
$$

hence formula (12) will be changed into

$$
\Phi_{\theta_{1}}^{2}=\Phi^{4}+p \Phi^{2}+q \Phi+r
$$

where

$$
\begin{aligned}
& p=\frac{a_{2}-\frac{3}{2} b_{1}^{2} a_{4}}{\sqrt{a_{4}}}, \\
& q=\frac{a_{1}+a_{4} b_{1}^{3}-a_{2} b_{1}}{\sqrt[4]{a_{4}}}, \\
& r=a_{0}-\frac{1}{2} a_{1} b_{1}+\frac{1}{4} a_{2} b_{1}{ }^{2}-\frac{3}{16} a_{4} b_{1}{ }^{4} .
\end{aligned}
$$

Then we can obtain

$$
\pm\left(\theta_{1}-\theta_{0}\right)=\int \frac{d \Phi}{\sqrt{\Phi^{4}+p \Phi^{2}+q \Phi+r}} .
$$

According to formula (14), the complete discrimination system is presented as

$$
\begin{aligned}
& D_{1}=4, \quad D_{2}=-p, \quad D_{3}=-2 p^{3}+8 p r-9 q^{2}, \quad E_{2}=9 p^{2}-32 p r, \\
& D_{4}=-p^{3} q^{2}+4 p^{4} r+36 p q^{2}-32 p^{2} r^{2}-\frac{27}{4} q^{4}+64 r^{3} .
\end{aligned}
$$


In order to solve formula (18), the solutions will be demonstrated in nine cases.

Case 1. $D_{2}=0, D_{3}=0$, and $D_{4}=0 . \Phi_{\theta_{1}}^{2}$ has a root of multiplicities four

$$
\Phi_{\theta_{1}}^{2}=\Phi^{4}
$$

Therefore, by using formula (18), we can get

$$
\begin{aligned}
& \left(\theta_{1}-\theta_{0}\right)=-\Phi^{-1}, \\
& \Phi=-\left(\theta_{1}-\theta_{0}\right)^{-1} .
\end{aligned}
$$

Thus the solutions of formula (12) are expressed as follows:

$$
\Upsilon(\theta)=\mp a_{4}^{-\frac{1}{4}}\left(a_{4}^{\frac{1}{4}} \theta-\theta_{0}\right)^{-1}-\frac{b_{1}}{2}
$$

For example, when $c=1, \kappa=1, \mu=-6, \delta=-6, c_{0}=0, c_{1}=0$, the solution of formula (3) is

$$
u(x, t)=-\left(\frac{x^{\alpha}}{\Gamma(1+\alpha)}-\frac{t^{\alpha}}{\Gamma(1+\alpha)}-\theta_{0}\right)^{-1}-\frac{1}{2}
$$

Case 2. $D_{2}<0, D_{3}=0, D_{4}=0 . \Phi_{\theta_{1}}^{2}$ has a pair of conjugate complex roots of multiplicities two:

$$
\Phi_{\theta_{1}}^{2}=\left((\Phi-l)^{2}+m^{2}\right)^{2}
$$

where $m>0$. By using formula (18), we attain

$$
\left(\theta_{1}-\theta_{0}\right)=\frac{1}{m} \arctan \frac{\Phi-l}{m}
$$

then the solution of formula (18) can be derived as

$$
\Phi=m \tan m\left(\theta_{1}-\theta_{0}\right)+l
$$

When $r=\frac{p^{2}}{4}, q=0, p>0$, then $m=\frac{p}{2}$, the solutions of formula (12) are presented as follows:

$$
\Upsilon(\theta)= \pm a_{4}^{-\frac{1}{4}} m \tan \left(m\left(a_{4}^{\frac{1}{4}} \theta-\theta_{0}\right)\right)-\frac{b_{1}}{2}
$$

For instance, when $c=\frac{7}{2}, \kappa=1, \mu=-6, \delta=-6, c_{0}=\frac{5}{4}, c_{1}=-\frac{25}{32}$, what we attain is as follows:

$$
u(x, t)=\tan \left(\frac{x^{\alpha}}{\Gamma(1+\alpha)}-\frac{7 t^{\alpha}}{2 \Gamma(1+\alpha)}-\theta_{0}\right)-\frac{1}{2}
$$

Case 3. $D_{2}>0, D_{3}=0, D_{4}=0, E_{2}=0 . \Phi_{\theta_{1}}^{2}$ has a real root of multiplicities three and a real root of multiplicity one

$$
\Phi_{\theta_{1}}^{2}=(\Phi-m)(\Phi-l)^{3}
$$


Using formula (18) can yield

$$
\pm\left(\theta_{1}-\theta_{0}\right)=\frac{2}{m-l} \sqrt{\frac{\Phi-m}{\Phi-l}} .
$$

When $\Phi>m, \Phi>l$ or $\Phi<m, \Phi<l$, the solution of formula (18) is

$$
\Phi=\frac{4(l-m)}{\left(\theta_{1}-\theta_{0}\right)^{2}(m-l)^{2}-4}+l
$$

Formula (32) has the rational function solution. The solution of formula (12) gotten by us is

$$
\Upsilon(\theta)= \pm \frac{4 a_{4}^{-\frac{1}{4}}(l-m)}{\left(a_{4}^{\frac{1}{4}} \theta-\theta_{0}\right)^{2}(m-l)^{2}-4}-\frac{b_{1}}{2}+l .
$$

For example, when $c=-6, \kappa=1, \delta=-6, \mu=-6, c_{0}=-4, c_{1}=-\frac{3}{2}$, the solution of formula (3) is expressed as follows:

$$
u(x, t)=\frac{4}{4\left(\frac{x^{\alpha}}{\Gamma(1+\alpha)}+\frac{6 t^{\alpha}}{\Gamma(1+\alpha)}-\theta_{0}\right)^{2}-1}+\frac{1}{2} .
$$

Case 4. $D_{2}>0, D_{3}=0, D_{4}=0, E_{2}>0 . \Phi_{\theta_{1}}^{2}$ has two real roots of multiplicities two, namely

$$
\Phi_{\theta_{1}}^{2}=(\Phi-m)^{2}(\Phi-l)^{2}
$$

we have

$$
\pm\left(\theta_{1}-\theta_{0}\right)=\frac{1}{l-m} \ln \left|\frac{\Phi-l}{\Phi-m}\right| .
$$

The solution of formula (12) is given by

$$
\Upsilon(\theta)=\frac{(m-l) a_{4}^{-\frac{1}{4}}}{2}\left[\operatorname{coth} \frac{(l-m)\left(a_{4}^{\frac{1}{4}} \theta-\theta_{0}\right)}{2}-1\right]-\frac{b_{1}}{2}+m
$$

When $m<\Phi<l$, we get the solution as follows:

$$
\Phi=\frac{m-l}{2}\left[\tanh \frac{(l-m)\left(\theta_{1}-\theta_{0}\right)}{2}-1\right]+m .
$$

Similarly,

$$
\Upsilon(\theta)=\left[\tanh \frac{(l-m)\left(a_{4}^{\frac{1}{4}} \theta-\theta_{0}\right)}{2}-1\right] \frac{(m-l) a_{4}^{-\frac{1}{4}}}{2}-\frac{b_{1}}{2}+m,
$$

i.e., $r=\frac{p^{2}}{4}, q=0, p<0$, then $m=-\sqrt{-p}, l=\sqrt{-p}$. For instance, when $\kappa=1, c=-\frac{1}{2}, \delta=-6$, $\mu=-6, c_{0}=-\frac{3}{4}, c_{1}=\frac{9}{32}$, and $\Phi>1$ or $\Phi<-1$, we can get

$$
u(x, t)=-\operatorname{coth}\left(\frac{x^{\alpha}}{\Gamma(1+\alpha)}+\frac{t^{\alpha}}{2 \Gamma(1+\alpha)}-\theta_{0}\right)-\frac{3}{2} .
$$


Case 5. When $D_{4}>0$ and $D_{2}>0, D_{3}>0$,

$$
\Phi_{\theta_{1}}^{2}=\prod_{i=1}^{4}\left(\Phi-\alpha_{i}\right)
$$

where $\alpha_{i}(i=1,2,3,4)$ are real numbers and $\alpha_{i}$ in turn decrease. If $\Phi>\alpha_{1}$ or $\Phi<\alpha_{4}$, then the transformation is as follows:

$$
\Phi=\frac{\alpha_{2}\left(\alpha_{1}-\alpha_{4}\right) \sin ^{2} \vartheta-\alpha_{1}\left(\alpha_{2}-\alpha_{4}\right)}{\left(\alpha_{1}-\alpha_{4}\right) \sin ^{2} \vartheta-\left(\alpha_{2}-\alpha_{4}\right)}
$$

if $\alpha_{3}<\Phi<\alpha_{2}$, similarly

$$
\Phi=\frac{\alpha_{4}\left(\alpha_{2}-\alpha_{3}\right) \sin ^{2} \vartheta-\alpha_{3}\left(\alpha_{2}-\alpha_{4}\right)}{\left(\alpha_{2}-\alpha_{3}\right) \sin ^{2} \vartheta-\left(\alpha_{2}-\alpha_{4}\right)}
$$

Combining formula (42) or formula (43) with formula (18), we can have

$$
\theta_{1}-\theta_{0}=\frac{2}{\sqrt{\left(\alpha_{1}-\alpha_{3}\right)\left(\alpha_{2}-\alpha_{4}\right)}} \int \frac{d \vartheta}{\sqrt{1-m^{2} \sin ^{2} \vartheta}},
$$

where $m^{2}=\frac{\left(\alpha_{1}-\alpha_{4}\right)\left(\alpha_{2}-\alpha_{3}\right)}{\left(\alpha_{1}-\alpha_{3}\right)\left(\alpha_{2}-\alpha_{4}\right)}$, based on Jacobian elliptic sine function [38] and formula (45), we have

$$
\sin \vartheta=\operatorname{sn}\left(\left(\theta_{1}-\theta_{0}\right) \frac{\sqrt{\left(\alpha_{1}-\alpha_{3}\right)\left(\alpha_{2}-\alpha_{4}\right)}}{2}, m\right) .
$$

Combining formula (45) with formula (42) and (43), the solutions of formula (18) with corresponding conditions are as follows:

$$
\Phi=\frac{\operatorname{sn}^{2}\left(\frac{\sqrt{\left(\alpha_{1}-\alpha_{3}\right)\left(\alpha_{2}-\alpha_{4}\right)}}{2} \alpha_{2}\left(\alpha_{1}-\alpha_{4}\right)\left(\theta_{1}-\theta_{0}\right), m\right)-\alpha_{1}\left(\alpha_{2}-\alpha_{4}\right)}{\operatorname{sn}^{2}\left(\frac{\sqrt{\left(\alpha_{1}-\alpha_{3}\right)\left(\alpha_{2}-\alpha_{4}\right)}}{2}\left(\alpha_{1}-\alpha_{4}\right)\left(\theta_{1}-\theta_{0}\right), m\right)-\left(\alpha_{2}-\alpha_{4}\right)},
$$

then we have

$$
\Upsilon(\theta)=\frac{\operatorname{sn}^{2}\left(\frac{\sqrt{\left(\alpha_{1}-\alpha_{3}\right)\left(\alpha_{2}-\alpha_{4}\right)}}{2} \alpha_{4}\left(\alpha_{2}-\alpha_{3}\right) a_{4}^{-\frac{1}{4}}\left(a_{4}^{\frac{1}{4}} \theta-\theta_{0}\right), m\right)-\alpha_{3}\left(\alpha_{2}-\alpha_{4}\right)}{\operatorname{sn}^{2}\left(\frac{\sqrt{\left(\alpha_{1}-\alpha_{3}\right)\left(\alpha_{2}-\alpha_{4}\right)}}{2}\left(\alpha_{2}-\alpha_{3}\right)\left(a_{4}^{\frac{1}{4}} \theta-\theta_{0}\right), m\right)-\left(\alpha_{2}-\alpha_{4}\right)}-\frac{b_{1}}{2},
$$

where $m^{2}=\frac{\left(\alpha_{1}-\alpha_{4}\right)\left(\alpha_{2}-\alpha_{3}\right)}{\left(\alpha_{1}-\alpha_{3}\right)\left(\alpha_{2}-\alpha_{4}\right)}$. Formulas (46) and (47) are elliptic functions double periodic solutions such as, when $c=-\frac{7}{2}, \kappa=1, \mu=-6, \delta=-6, c_{0}=-\frac{9}{4}, c_{1}=\frac{45}{32}$, we have $\alpha_{1}=2$, $\alpha_{2}=1, \alpha_{3}=-1, \alpha_{4}=-2$, if $\Phi>\alpha_{1}$ or $\Phi<\alpha_{4}$, the solution obtained by us is

$$
u(x, t)=\frac{4 \operatorname{sn}^{2}\left(\frac{3}{2}\left(\frac{x^{\alpha}}{\Gamma(1+\alpha)}+\frac{7 t^{\alpha}}{2 \Gamma(1+\alpha)}-\theta_{0}\right), \frac{2 \sqrt{2}}{3}\right)-6}{4 \operatorname{sn}^{2}\left(\frac{3}{2}\left(\frac{x^{\alpha}}{\Gamma(1+\alpha)}+\frac{7 t^{\alpha}}{2 \Gamma(1+\alpha)}-\theta_{0}\right), \frac{2 \sqrt{2}}{3}\right)-3}-\frac{1}{2}
$$

Case 6. When $D_{2} D_{3}<0$ and $D_{4}=0, \Phi_{\theta_{1}}^{2}$ has a real root of multiplicities two and a pair of conjugate complex roots:

$$
\Phi_{\theta_{1}}^{2}=(\Phi-\beta)^{2}\left[m^{2}+(\Phi-l)^{2}\right]
$$


where $m, l$, and $\beta$ are real numbers. According to formula (18), we have

$$
\pm\left(\theta_{1}-\theta_{0}\right)=\frac{1}{\sqrt{m^{2}+(\beta-l)^{2}}} \ln \left|\frac{\nu \Phi+\eta-\sqrt{\left(m^{2}+\Phi-l\right)^{2}}}{\Phi-\beta}\right|
$$

where

$$
v=\frac{\beta-2 l}{\sqrt{(\beta-l)^{2}+m^{2}}}, \quad \eta=\sqrt{(\beta-l)^{2}+m^{2}}-\frac{\beta(\beta-2 l)}{\sqrt{(\beta-l)^{2}+m^{2}}}
$$

and then we can get the solution of formula (12):

$$
\Upsilon(\theta)=\frac{\left(e^{ \pm \sqrt{(\beta-l)^{2}+m^{2}}\left(a_{4}^{\frac{1}{4}} \theta-\theta_{0}\right)}-\epsilon\right)+\sqrt{(\beta-l)^{2}+m^{2}}(2-\epsilon)}{a_{4}^{\frac{1}{4}}\left[\left(e^{ \pm \sqrt{(\beta-l)^{2}+m^{2}}\left(a_{4}^{\frac{1}{4}} \theta-\theta_{0}\right)}-\epsilon\right)^{2}-1\right]}-\frac{b_{1}}{2},
$$

$\Upsilon(\theta)$ has a solitary wave solution. When $c=\frac{7}{2}, \kappa=1, \delta=-6, \mu=-6, c_{0}=\frac{21}{4}, c_{1}=\frac{153}{32}$, we can obtain the solution of formula (3) as follows:

$$
u(x, t)=\frac{e^{ \pm 2 \sqrt{2}\left(\frac{x^{\alpha}}{\Gamma(1+\alpha)}-\frac{7 t^{\alpha}}{2 \Gamma(1+\alpha)}-\theta_{0}\right)}+\frac{13 \sqrt{2}}{4}-3}{\left[\left(e^{ \pm 2 \sqrt{2}\left(\frac{x^{\alpha}}{\Gamma(1+\alpha)}-\frac{7 t^{\alpha}}{2 \Gamma(1+\alpha)}-\theta_{0}\right)}-\frac{3 \sqrt{2}}{4}\right)^{2}-1\right]}-\frac{1}{2}
$$

Case 7. When $D_{4}<0$ and $D_{2} D_{3} \geq 0, \Phi_{\theta_{1}}^{2}$ has two distinct real roots and a pair of conjugate complex roots, then $\Phi_{\theta_{1}}^{2}$ is given by

$$
\Phi_{\theta_{1}}^{2}=\left[(\Phi-l)^{2}+m^{2}\right](\Phi-\gamma)(\Phi-\beta)
$$

where $m, l, \gamma$, and $\beta$ are real numbers, $m>0$ and $\beta>\gamma$. The following transformation is

$$
\Phi=\frac{d_{1} \cos \vartheta+d_{2}}{d_{3} \cos \vartheta+d_{4}}
$$

where

$$
\begin{aligned}
& d_{1}=\frac{1}{2}(\beta+\gamma) d_{3}-\frac{1}{2}(\beta-\gamma) d_{4}, \\
& d_{2}=\frac{1}{2}(\beta+\gamma) d_{4}-\frac{1}{2}(\beta-\gamma) d_{3}, \\
& d_{3}=\beta-l-\frac{m}{f_{2}} \\
& d_{4}=\beta-l-m f_{2}, \\
& e_{2}=\frac{m^{2}+(\gamma-l)(\beta-l)}{m(\beta-\gamma)}, \\
& f_{2}=e_{2} \pm \sqrt{e_{2}^{2}+1} .
\end{aligned}
$$


We choose the sign of $f_{2}$ such that $f_{2}>0$. Combining formula (55) with formula (18), we have

$$
\theta_{1}-\theta_{0}=\frac{2 f_{2} m_{2}}{\sqrt{\mp 2 m f_{2}(\beta-\gamma)}} \int \frac{d \Phi}{\sqrt{1-m_{2}^{2} \sin ^{2} \vartheta}}
$$

where $m_{2}^{2}=\frac{2}{1+f_{2}^{2}}$. According to formula (57) and Jacobian elliptic cosine function [38], we have

$$
\cos \vartheta=\operatorname{cn}\left(\frac{\sqrt{\mp 2 m f_{2}(\beta-\gamma)}}{2 f_{2} m_{2}}\left(\theta_{1}-\theta_{0}\right), m_{2}\right) .
$$

Combining formula (58) with formula (55), we can obtain the solutions of formula (18) as follows:

$$
\Phi=\frac{d_{1} \operatorname{cn}\left(\frac{\sqrt{\mp 2 m f_{2}(\beta-\gamma)}}{2 f_{2} m_{2}}\left(\theta_{1}-\theta_{0}\right), m_{2}\right)+d_{2}}{d_{3} \operatorname{cn}\left(\frac{\sqrt{\mp 2 m f_{2}(\beta-\gamma)}}{2 f_{2} m_{2}}\left(\theta_{1}-\theta_{0}\right), m_{2}\right)+d_{4}}
$$

hence the solution of formula (12) is given by

$$
\Upsilon(\theta)=\frac{a_{4}^{-\frac{1}{4}}\left[d_{1} \operatorname{cn}\left(\frac{\sqrt{\mp 2 m f_{2}(\beta-\gamma)}}{2 f_{2} m_{2}}\left(a_{4}^{\frac{1}{4}} \theta-\theta_{0}\right), m_{2}\right)+d_{2}\right]}{d_{3} \operatorname{cn}\left(\frac{\sqrt{\mp 2 m f_{2}(\beta-\gamma)}}{2 f_{2} m_{2}}\left(a_{4}^{\frac{1}{4}} \theta-\theta_{0}\right), m_{2}\right)+d_{4}}-\frac{b_{1}}{2}
$$

such as, when $c=\frac{11}{2}, \kappa=1, \delta=-6, \mu=-6, c_{0}=-\frac{9}{4}, c_{1}=-\frac{47}{32}$, we have $d_{1}=3, d_{2}=d_{3}=0$, $d_{4}=-3, e_{2}=\frac{3}{4}, f_{2}=2$, we can obtain the solution of formula (3):

$$
u(x, t)=-\operatorname{cn}\left(\frac{\sqrt{10}}{2}\left(\frac{x^{\alpha}}{\Gamma(1+\alpha)}-\frac{11 t^{\alpha}}{2 \Gamma(1+\alpha)}-\theta_{0}\right), \frac{\sqrt{10}}{5}\right)-\frac{1}{2}
$$

Case 8 . When $D_{4}>0$ and $D_{2} D_{3} \leq 0, \Phi_{\theta_{1}}^{2}$ has two pairs of conjugate complex roots:

$$
\Phi_{\theta_{1}}^{2}=\left(\left(\Phi-\alpha_{1}\right)^{2}+l_{1}^{2}\right)\left(\left(\Phi-\alpha_{2}\right)^{2}+l_{2}^{2}\right)
$$

where $l_{1}, l_{2}, \alpha_{1}$, and $\alpha_{2}$ are real numbers, $l_{1} \geq l_{2}>0$. The transformation is as follows:

$$
\Phi=\frac{d_{1} \tan \vartheta+d_{2}}{d_{3} \tan \vartheta+d_{4}}
$$


where

$$
\begin{aligned}
& d_{1}=\alpha_{1} d_{3}+l_{1} d_{4}, \\
& d_{2}=\alpha_{1} d_{4}-l_{1} d_{3}, \\
& d_{3}=-l_{1}-\frac{l_{2}}{f_{2}}, \\
& d_{4}=\alpha_{1}-\alpha_{2}, \\
& e_{2}=\frac{\left(\alpha_{1}-\alpha_{2}\right)^{2}+l_{1}^{2}+l_{2}^{2}}{2 l_{1} l_{2}}, \\
& f_{2}=e_{2}+\sqrt{e_{2}^{2}-1},
\end{aligned}
$$

which yields

$$
\theta_{1}-\theta_{0}=\frac{d_{3}^{2}+d_{4}^{2}}{l_{2} \sqrt{\left(d_{3}^{2}+d_{4}^{2}\right)\left(f_{2}^{2} d_{3}^{2}+d_{4}^{2}\right)}} \int \frac{d \vartheta}{\sqrt{1-m_{2}^{2} \sin ^{2} \vartheta}}
$$

where $m_{2}^{2}=\frac{f_{2}^{2}-1}{f_{2}^{2}}$. Based on the Jacobian elliptic function [38] and formula (63), we obtain

$$
\begin{aligned}
& \sin \vartheta=\operatorname{sn}\left(\frac{l_{2} \sqrt{\left(d_{3}^{2}+d_{4}^{2}\right)\left(f_{2}^{2} d_{3}^{2}+d_{4}^{2}\right)}}{d_{3}^{2}+d_{4}^{2}}\left(\theta_{1}-\theta_{0}\right), m_{2}\right), \\
& \cos \vartheta=\operatorname{cn}\left(\frac{l_{2} \sqrt{\left(d_{3}^{2}+d_{4}^{2}\right)\left(f_{2}^{2} d_{3}^{2}+d_{4}^{2}\right)}}{d_{3}^{2}+d_{4}^{2}}\left(\theta_{1}-\theta_{0}\right), m_{2}\right) .
\end{aligned}
$$

Combining formula (66) and formula (67) with formula (63), we have

$$
\Phi=\frac{d_{1} \operatorname{sn}\left(\eta\left(\theta_{1}-\theta_{0}\right), m_{2}\right)+d_{2} \operatorname{cn}\left(\eta\left(\theta_{1}-\theta_{0}\right), m_{2}\right)}{d_{3} \operatorname{sn}\left(\eta\left(\theta_{1}-\theta_{0}\right), m_{2}\right)+d_{4} \operatorname{cn}\left(\eta\left(\theta_{1}-\theta_{0}\right), m_{2}\right)}
$$

and

$$
\Upsilon(\theta)=\frac{d_{1} a_{4}^{-\frac{1}{4}} \operatorname{sn}\left(\eta\left(a_{4}^{\frac{1}{4}} \theta-\theta_{0}\right), m_{2}\right)+d_{2} \operatorname{cn}\left(\eta\left(a_{4}^{\frac{1}{4}} \theta-\theta_{0}\right), m_{2}\right)}{d_{3} \operatorname{sn}\left(\eta\left(a_{4}^{\frac{1}{4}} \theta-\theta_{0}\right), m_{2}\right)+d_{4} \operatorname{cn}\left(\eta\left(a_{4}^{\frac{1}{4}} \theta-\theta_{0}\right), m_{2}\right)}-\frac{b_{1}}{2},
$$

where

$$
\eta=\frac{l_{2} \sqrt{\left(d_{3}^{2}+d_{4}^{2}\right)\left(f_{2}^{2} d_{3}^{2}+d_{4}^{2}\right)}}{d_{3}^{2}+d_{4}^{2}}
$$

Formula (69) is an elliptic functions double periodic solution. When $c=11, \kappa=1, \delta=-6$, $\mu=-6, c_{0}=\frac{5 \sqrt{7}-10}{2}, c_{1}=\frac{253-10 \sqrt{7}}{8}$, we have $\alpha_{1}=\frac{\sqrt{7}}{2}, \alpha_{2}=-\frac{\sqrt{7}}{2}, l_{1}=3, l_{2}=2$, and $d_{1}=\frac{7 \sqrt{7}}{6}$, $d_{2}=\frac{29}{2}, d_{3}=-\frac{11}{3}, d_{4}=\sqrt{7}, e_{2}=\frac{5}{3}, f_{2}=3$, and $\eta=\frac{24 \sqrt{23}}{23}$, then

$$
u(x, t)=\frac{\frac{7 \sqrt{7}}{6} \operatorname{sn}\left(\frac{24 \sqrt{23}}{23}\left(\frac{x^{\alpha}}{\Gamma(1+\alpha)}-\frac{11 t^{\alpha}}{\Gamma(1+\alpha)}-\theta_{0}\right), \frac{2 \sqrt{2}}{3}\right)+\frac{29}{2} \operatorname{cn}\left(\frac{24 \sqrt{23}}{23}\left(\frac{x^{\alpha}}{\Gamma(1+\alpha)}-\frac{11 t^{\alpha}}{\Gamma(1+\alpha)}-\theta_{0}\right), \frac{2 \sqrt{2}}{3}\right)}{-\frac{11}{3} \operatorname{sn}\left(\frac{24 \sqrt{23}}{23}\left(\frac{x^{\alpha}}{\Gamma(1+\alpha)}-\frac{11 t^{\alpha}}{\Gamma(1+\alpha)}-\theta_{0}\right), \frac{2 \sqrt{2}}{3}\right)+\sqrt{7} \operatorname{cn}\left(\frac{24 \sqrt{23}}{23}\left(\frac{x^{\alpha}}{\Gamma(1+\alpha)}-\frac{11 t^{\alpha}}{\Gamma(1+\alpha)}-\theta_{0}\right), \frac{2 \sqrt{2}}{3}\right)}
$$




$$
-\frac{1}{2}
$$

Case 9. When $D_{2}>0, D_{3}>0$, and $D_{4}=0, \Phi_{\theta_{1}}^{2}$ has two single real roots and a real root with multiplicities two:

$$
\Phi_{\theta_{1}}^{2}=\left(\Phi-\alpha_{1}\right)^{2}\left(\Phi-\alpha_{2}\right)\left(\Phi-\alpha_{3}\right)
$$

where $\alpha_{i}(i=1,2,3,4)$ are real numbers, and $\alpha_{2}>\alpha_{3}, \alpha_{1}=-\frac{\alpha_{2}+\alpha_{3}}{2}$. Denoting $h=\left(\alpha_{1}-\right.$ $\left.\alpha_{2}\right)\left(\alpha_{1}-\alpha_{3}\right)$, when $\Phi>\alpha_{2}, \alpha_{2}>\alpha_{1}>\alpha_{3}$, we can get the solution of formula (18) as follows:

$$
\Phi=\frac{2 h}{ \pm\left(\alpha_{2}-\alpha_{3}\right) \sin \left[\sqrt{-h}\left(\theta_{1}-\theta_{0}\right)\right]-\left(2 \alpha_{1}-\alpha_{2}-\alpha_{3}\right)},
$$

then we can have

$$
\Upsilon(\theta)=\frac{2 a_{4}^{-\frac{1}{4}} h}{ \pm\left(\alpha_{2}-\alpha_{3}\right) \sin \left[\sqrt{-h}\left(a_{4}^{\frac{1}{4}} \theta-\theta_{0}\right)\right]-\left(2 \alpha_{1}-\alpha_{2}-\alpha_{3}\right)}-\frac{b_{1}}{2},
$$

when $\alpha_{1}>\alpha_{2}$ or $\alpha_{1}<\alpha_{3}$,

$$
\Phi=\frac{2 h}{\left(\alpha_{2}-\alpha_{3}\right) \cosh \left[\sqrt{h}\left(\theta_{1}-\theta_{0}\right)\right]-\left(2 \alpha_{1}-\alpha_{2}-\alpha_{3}\right)},
$$

we can obtain

$$
\Upsilon(\theta)=\frac{2 a_{4}^{-\frac{1}{4}} h}{\left(\alpha_{2}-\alpha_{3}\right) \cosh \left[\sqrt{h}\left(a_{4}^{\frac{1}{4}} \theta-\theta_{0}\right)\right]-\left(2 \alpha_{1}-\alpha_{2}-\alpha_{3}\right)}-\frac{b_{1}}{2} .
$$

For instance, $\kappa=1, c=-\frac{25}{2}, \delta=-6, \mu=6, c_{0}=\frac{63}{4}, c_{1}=-\frac{327}{32}$, and then

$$
u(x, t)=\frac{-10}{\sinh \left[\sqrt{5}\left(\frac{x^{\alpha}}{\Gamma(1+\alpha)}+\frac{25^{\alpha}}{2 \Gamma(1+\alpha)}-\theta_{0}\right)\right]-4}+\frac{1}{2},
$$

which is a solitary wave solution.

\section{Numerical simulations}

In this section, numerical simulations of space-time fractional KdV-mKdV equation are given. In order to see the results more intuitively, based on the solutions that we got above, different types of solutions are selected for numerical simulation, including rational function solutions to formulas (24) and (34), trigonometric functional periodic solutions to formulas (29) and (40), Jacobian elliptic functions with double periodic solutions to formulas (48), (61), and (71), solitary wave solutions to formulas (53) and (77). The properties of the solution are expressed by drawing a three-dimensional figure and the corresponding two-dimensional figure. One thing to note is that we only concentrate on the positive if there is a plus or minus sign in the selected solution.

Case 1. For $\alpha=0.7$ (Fig. 1). 


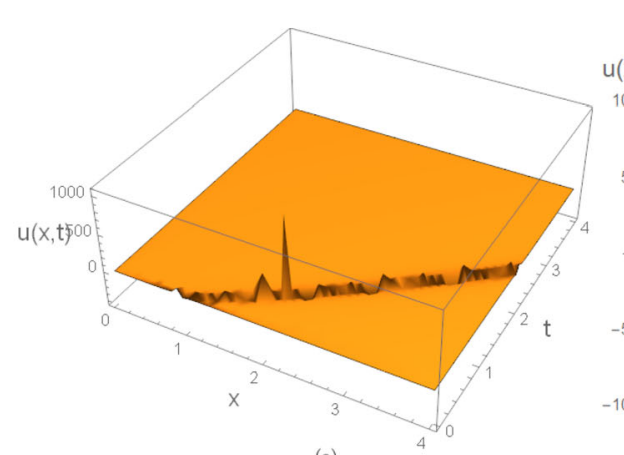

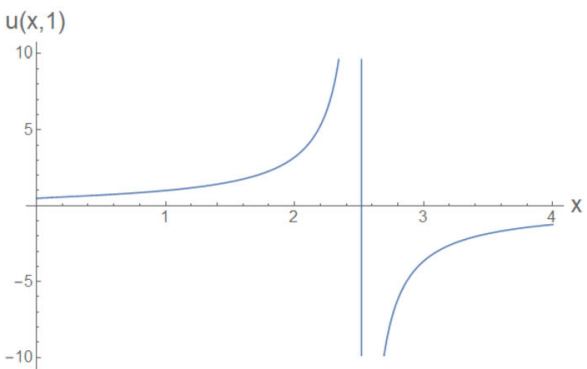

Figure 1 (a) Three-dimensional figure of (24), when $\kappa=1, c=1$, and $\xi_{0}=1$; (b) the corresponding two-dimensional figure when $t=1$

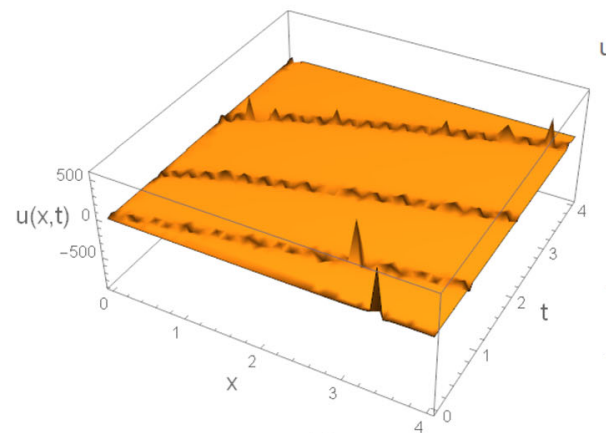

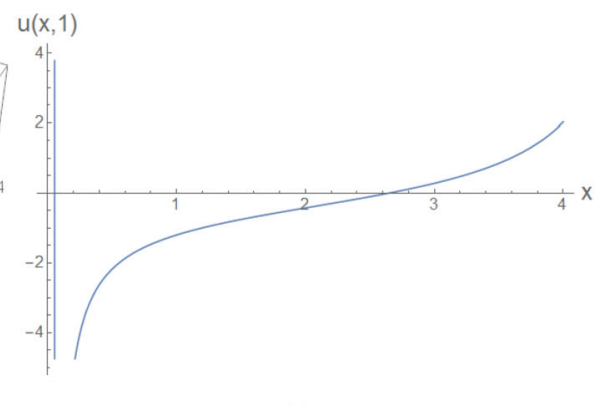

Figure 2 (a) Three-dimensional figure of (29), when $\kappa=1, c=\frac{7}{2}$, and $\xi_{0}=1$; (b) the corresponding two-dimensional figure when $t=1$

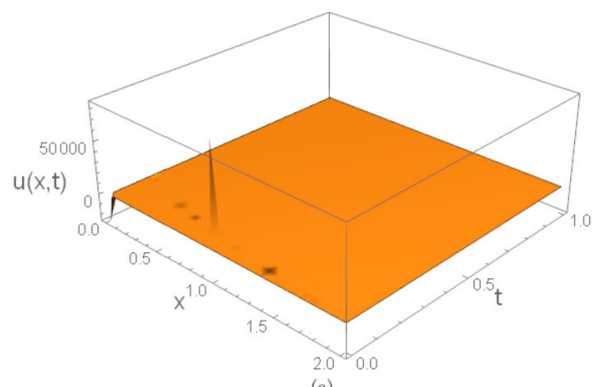

(a)

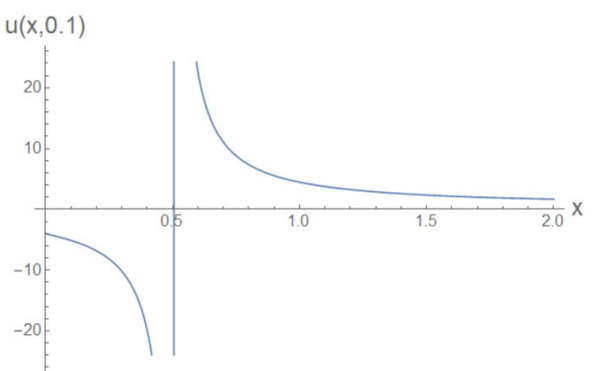

(b)

Figure 3 (a) Three-dimensional figure of (34), when $\kappa=1, c=-6$, and $\xi_{0}=1$; (b) the corresponding two-dimensional figure when $t=0.1$

Case 2. For $\alpha=0.7$ (Fig. 2).

Case 3. For $\alpha=0.7$ (Fig. 3).

Case 4. For $\alpha=0.7$ (Fig. 4).

Case 5. For $\alpha=0.7$ (Fig. 5).

Case 6. For $\alpha=0.7$ (Fig. 6).

Case 7. For $\alpha=0.7$ (Fig. 7). 


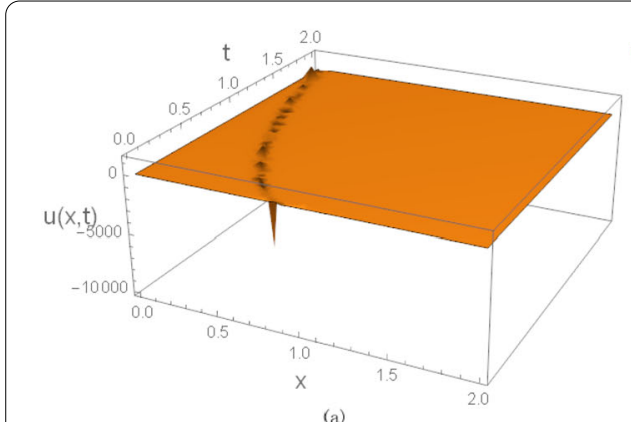

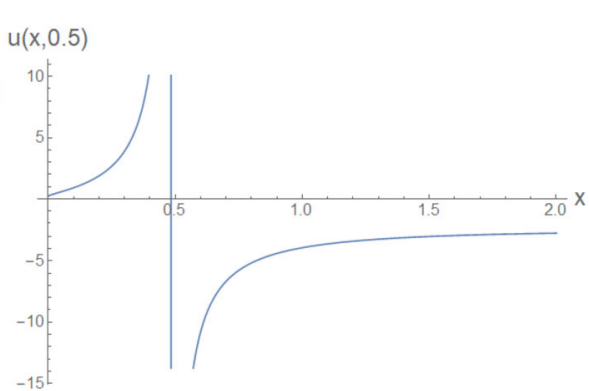

Figure 4 (a) Three-dimensional figure of (40), when $\kappa=1, c=-\frac{1}{2}$, and $\xi_{0}=1$; (b) the corresponding two-dimensional figure when $t=0.5$

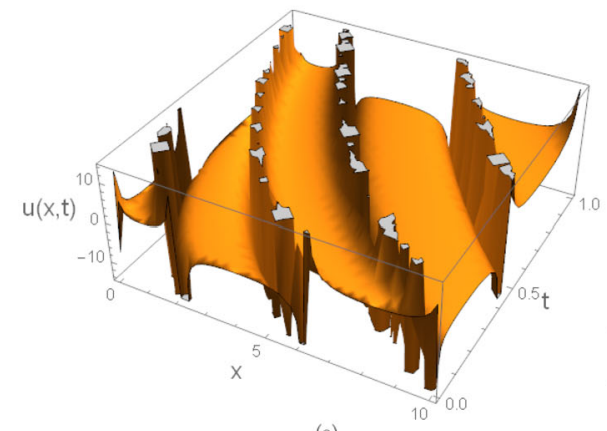

(a)

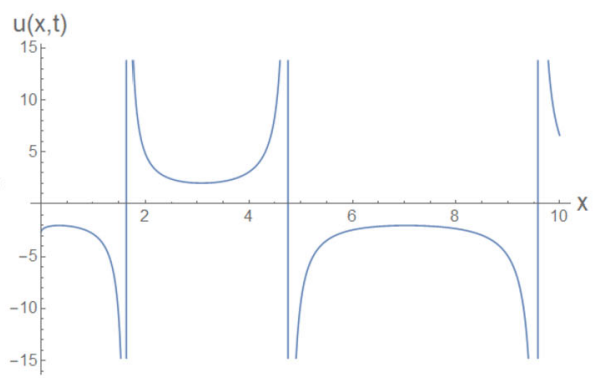

(b)

Figure 5 (a) Three-dimensional figure of (48), when $\kappa=1, c=-\frac{7}{2}$, and $\xi_{0}=1$; (b) the corresponding two-dimensional figure when $t=0.5$

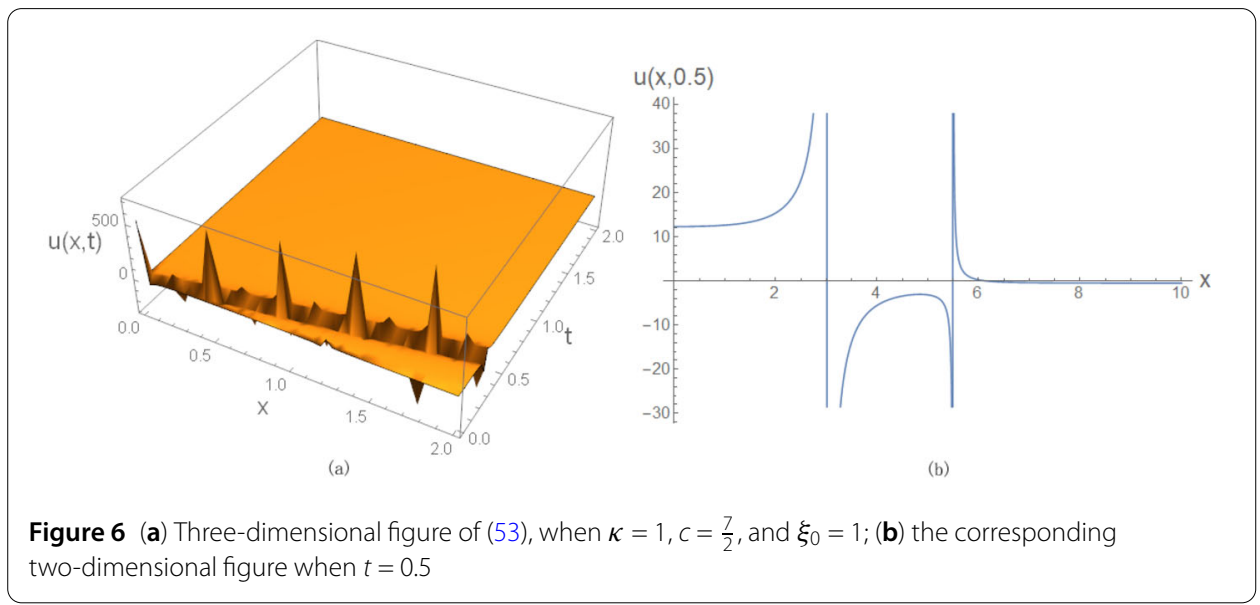

Case 8. For $\alpha=0.7$ (Fig. 8).

Case 9. For $\alpha=0.7$ (Fig. 9).

\section{Conclusion}

On the basis of traffic flow following theory and previous research work, this paper studies various nonlinear density wave problems of traffic flow in order to make our solutions more universal, considers space-time fractional $\mathrm{KdV}$-mKdV equation. It is difficult to ob- 


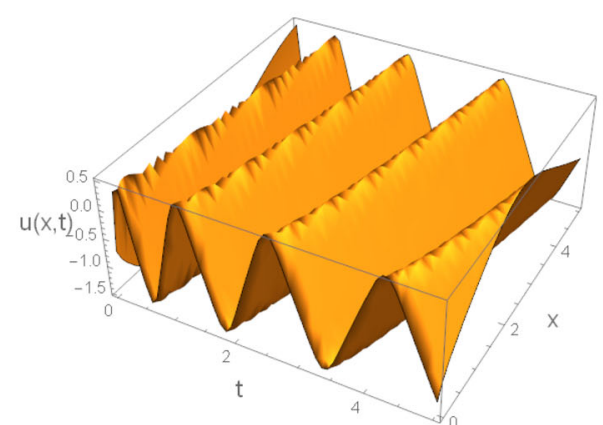

(a)

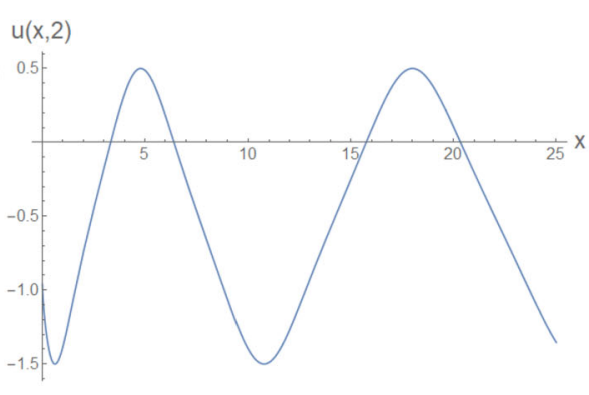

Figure 7 (a) Three-dimensional figure of (61), when $\kappa=1, c=\frac{11}{2}$, and $\xi_{0}=1$; (b) the corresponding two-dimensional figure when $t=2$

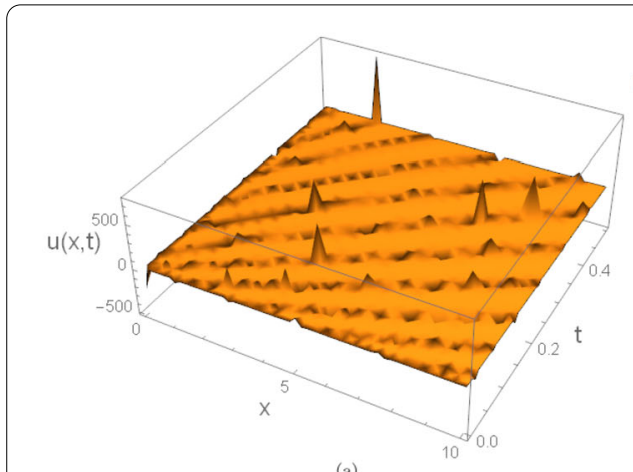

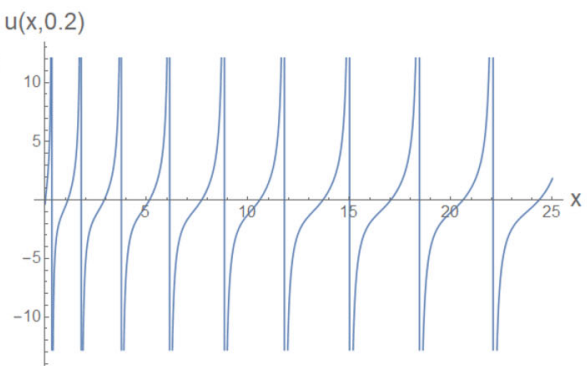

(b)

Figure 8 (a) Three-dimensional figure of (71), when $\kappa=1, c=11$, and $\xi_{0}=1$; (b) the corresponding two-dimensional figure when $t=0.2$

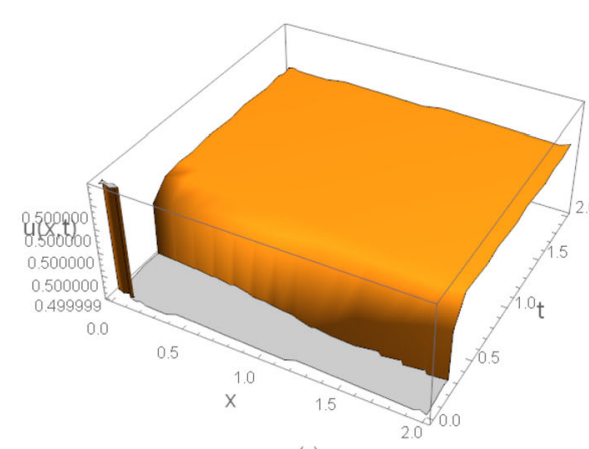

(a)

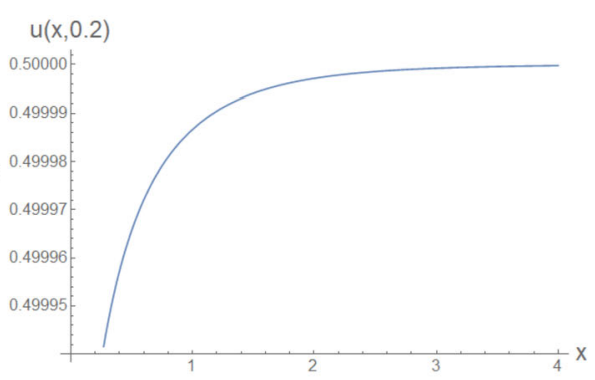

Figure 9 (a) Three-dimensional figure of (77), when $\kappa=1, c=-\frac{25}{2}$, and $\xi_{0}=1$; (b) the corresponding two-dimensional figure when $t=0.2$

tain Jacobian elliptic functions with periodic solutions by other methods. In this paper, the KdV-mKdV equation is transformed into space-time fractional equation, which is reduced to usual differential equations by using fractional complex transformation. All possible solutions are given by the complete discrimination system for polynomial method. Similar solutions have not been found in other literature, which also shows the strong role. 
In addition, in order to guarantee the existence of each solution, this paper sets specific parameters to get the solutions and the numerical simulation, also shows properties of the solutions.

\section{Acknowledgements}

The authors would like to thank the reviewers for their helpful comments and suggestions to improve the manuscript.

\section{Funding}

The paper was funded by the National Social Science Foundation of China (NO.15BJL104, NO.18BJL039).

\section{Availability of data and materials}

Data sharing not applicable to this article as no datasets were generated or analysed during the current study.

\section{Competing interests}

We would like to declare no conflict of interests.

\section{Authors' contributions}

$\mathrm{CL}, \mathrm{DC}$, and QD worked together in the derivation of the mathematical results. The authors read and approved the final manuscript.

\section{Publisher's Note}

Springer Nature remains neutral with regard to jurisdictional claims in published maps and institutional affiliations.

Received: 6 November 2019 Accepted: 4 February 2020 Published online: 11 February 2020

\section{References}

1. Boer, E.R.: Car following from the drive's perspective. Transp. Res., Part F 2, 201-206 (1999)

2. Kerner, B.S., Konhauser, P.: Cluster effect in initially homogeneous traffic flow. Phys. Rev. E 48(4), $2335-2338$ (1993)

3. Kerner, B.S., Klenov, S.L., Hiller, A.: Empirical test of a microscopic three-phase traffic theory. Nonlinear Dyn. 49(4), 525-553 (2007)

4. Ni, R., Kang, J.J., Andersen, J.G.: Age-related declines in car following performance under simulated fog conditions Accid. Anal. Prev. 42, 818-826 (2010)

5. Bando, M., Hasbe, K., Nakayama, A., Shibata, A., Sugiyama, Y.: Dynamics model of traffic congestion and numerical simulation. Phys. Rev. E 51(2), 1035-1042 (1995)

6. Helbing, D., Tilch, B.: Generalized force model of traffic dynamics. Phys. Rev. E 58(1), 133-138 (1998)

7. Peng, G.H., Cai, X.H., Liu, C.Q., Cao, B.F., Tuo, M.X.: Optimal velocity difference model for a car-following theory. Phys. Lett. A 375(45), 3973-3977 (2011)

8. Zhu, H.B., Dai, S.Q.: Numerical simulation of soliton and kink density waves in traffic flow with periodic boundaries. Physica A 387(16-17), 4367-4375 (2008)

9. Orosz, G., Wilson, R.E., Stepan, G.: Traffic jams: dynamics and control. Philos. Trans. R. Soc. Lond. A 368, 4455-4479 (2010)

10. Nagatani, T.: The physics of traffic jams. Rep. Prog. Phys. 65, 1331-1386 (2002)

11. Zheng, L.J., Tian, C., Sun, D.H., Liu, W.N.: A new car-following model with consideration of anticipation driving behavior. Nonlinear Dyn. 70(2), 1205-1211 (2012)

12. Li, Z.P., et al.: Study on the effects of driver's lane-changing aggressiveness on traffic stability from an extended two-lane lattice model. Commun. Nonlinear Sci. Numer. Simul. 24(1-3), 52-63 (2015)

13. Zhou, J., Shi, Z.K., Cao, J.L.: Nonlinear analysis of the optimal velocity difference model with reaction-time delay. Physica A 396, 77-87 (2014)

14. Hattam, L.: Travelling wave solutions of the perturbed $\mathrm{mKdV}$ equation that represent traffic congestion. Wave Motion 79, 57-72 (2018)

15. Shen, J.Y., Sun, Z.Z., Cao, W.R.: A finite difference scheme on graded meshes for time-fractional nonlinear Korteweg-deVries equation. Appl. Math. Comput. 361, 752-765 (2019)

16. Kaya, D.G., Gulbahar, S.M., Yokus, A., Gulbahar, M.: Solutions of the fractional combined KdV-mKdV equation with collocation method using radial basis function and their geometrical obstructions. Adv. Differ. Equ. 2018, 77 (2018)

17. Ma, W.X.: Long-time asymptotics of a three-component coupled mKdV system. Mathematics 7, 573 (2019)

18. Ablowitz, M.J., Clarkson, P.A.: Solitons, Nonlinear Evolutions and Inverse Scattering. Cambridge University Press, Cambridge (1991)

19. Gomez, C.A., Salas, A.H.: Exact solutions for the generalized BBM equation with variable coefficients. Math. Probl. Eng. 4(9), 394-401 (2010)

20. Yusufoglu, E., Bekir, A., Alp, M.: Periodic and solitary wave solutions of Kawahara and modified Kawahara equations by using sine-cosine method. Chaos Solitons Fractals 37, 1193-1197 (2008)

21. Wazwaz, A.M.: New travelling wave solutions of different physical structures to generalized BBM equation. Phys. Lett. A 355(4-5), 358-362 (2006)

22. Wadati, M.: Invariances and conservation laws of the Korteweg-de Vries equation. Stud. Appl. Math. 59(2), 59-153 (1978)

23. Bulman, G.W., Sukeyuki, K.: Symmetries and Differential Equations. Springer, New York (1991)

24. Komatsu, T.S., Sasa, S.: Kink soliton characterizing traffic congestion. Phys. Rev. E 52, 5574-5582 (1995)

25. Ma, W.X.: A search for lump solutions to a combined fourth-order nonlinear PDE in $(2+1)$-dimensions. J. Appl. Anal. Comput. 9, 1319-1332 (2019) 
26. Ma, W.X.: Interaction solutions to Hirota-Satsuma-Ito equation in (2+1)-dimensions. Front. Math. China 14,619-629 (2019)

27. Liu, C.S.: Classification of all single travelling wave solutions to Calogero-Degasperis-Focas equation. Commun. Theor. Phys. 48, 601-604 (2007)

28. Liu, C.S.: All single travelling wave solutions to Nizhnok-Novikov-Veselov equation. Commun. Theor. Phys. 45, 991-992 (2006)

29. Liu, C.S.: The classification of travelling wave solutions and superposition of multi-solutions to Camassa-Holm equation with dispersion. Chin. Phys. 16, 1832-1837 (2007)

30. Liu, C.S.: Representations and classifications of travelling wave solutions to sinh-Gordon equation. Commun. Theor. Phys. 49, 153-158 (2008)

31. Liu, C.S.: Solution of ODE $u^{\prime \prime}+p(u)\left(u^{\prime}\right)^{2}+q(u)=0$ and applications to classifications of all single travelling wave solutions to some nonlinear mathematical physics equations. Commun. Theor. Phys. 49, 291-296 (2008)

32. Liu, C.S.: Applications of complete discrimination system for polynomial for classifications of travelling wave solutions to nonlinear differential equations. Comput. Phys. Commun. 181, 317-324 (2010)

33. Ma, W.X., Fuchssteiner, B.: Explicit and exact solutions to a Kolmogorov-Petrovskii-Piskunov equation. Int. J. Non-Linear Mech. 31, 329-338 (1996)

34. Khalil, R., Horani, M.A., Yousef, A., et al.: A new definition of fractional derivative. J. Comput. Appl. Math. 264(5), 65-70 (2014)

35. Raslan, K.R., EL-Danaf, T.S., Khalid, K.: Aliexact solution of the space-time fractional coupled EW and coupled MEW equations. Eur. Phys. J. Plus 132, 319 (2017)

36. Ma, W.X., Lee, J.H.: A transformed rational function method and exact solutions to the $3+1$ dimensional Jimbo-Miwa equation. Chaos Solitons Fractals 42, 1356-1363 (2009)

37. Zhao, X.Q., Zhi, H.Y: An improved F-expansion method and its application to coupled Drinfel'd-Sokolov-Wilson equation. Commun. Theor. Phys. 50(2), 309 (2008)

38. Wang, Z.X., Guo, D.R.: Special Functions. Science Press, Beijing (2002)

\section{Submit your manuscript to a SpringerOpen ${ }^{\circ}$ journal and benefit from:}

- Convenient online submission

Rigorous peer review

- Open access: articles freely available online

- High visibility within the field

- Retaining the copyright to your article

Submit your next manuscript at $\gg$ springeropen.com 